\title{
METODOLOGÍA A+S EN PRÁCTICAS INICIALES: MODELO PEDAGÓGICO PARA LA FORMACIÓN POR COMPETENCIAS DE PSICÓLOGOS
}

\author{
Camila Astorga Olguín - Universidad Católica Silva Henríquez \\ Tatiana Soto López - Universidad Católica Silva Henríquez
}

Camila Astorga

Psicóloga, Especialización Organizacional UCSH

Magister en Liderazgo y Gestión Educativa, Universidad Diego Portales

Diploma en Competencias digitales para Enseñanza y el aprendizaje, CFP. Universidad de Sevilla Coordinadora Académica y Miembro de Comité curricular y pedagógico Departamento de Psicología UCSH

Tatiana Soto Psicóloga U. de Chile

Magister en Educación, Mención Política y Gestión Educacional, Universidad de la República Coordinadora de Vinculación con el Medio, Asesora en didáctica y Miembro de Comité curricular y pedagógico

Departamento de Psicología UCSH

\section{Correspondencia}

Camila Astorga - castorgao@ucsh.cl

Tatiana Soto - tsotol@ucsh.cl

General Jofré 462 Santiago de Chile - Región Metropolitana 


\title{
Resumen
}

El presente artículo da cuenta de un modelo de aplicación específico de la metodología Aprendizaje + Servicio diseñado y ejecutado por el Departamento de Psicología de la Universidad Católica Silva Henríquez en prácticas tempranas, constituyéndose en una propuesta pedagógica que busca vincular la teoría y la praxis desde el inicio de la formación para enriquecer el proceso de enseñanza-aprendizaje y el desarrollo de competencias disciplinares, sociales y personales de los futuros Psicólogos y Psicólogas. Posterior a una revisión teórico- conceptual para comprender la metodología $\mathrm{A}+\mathrm{S}$ y su relación con la formación por competencias y las habilidades para el siglo XXI, se aborda el modelo práctico utilizado por la carrera fundamentado en términos pedagógicos. Finalmente se invita a reflexionar acerca de la relevancia y los desafíos de incorporar en la formación de Psicología metodologías que potencien el desarrollo integral a través de experiencias que conecten al estudiante con la realidad social.

Palabras claves: aprendizaje y servicio; competencias; aprendizaje basado en proyectos; habilidades para el siglo XXI

\begin{abstract}
This article shows a specific application model of the Service + Learning methodology executed and designed by The Department of Psychology of Universidad Católica Silva Henríquez in early practices, becoming a pedagogical proposal that aims to link theory and praxis since the beginning of training to enrich teaching-learning process and the development of disciplinary, social and personal competences for the future Psychologists. A further theoretical-conceptual review to understand $\mathrm{S}+\mathrm{L}$ methodology and its involvement through training for competences and skills for the $21^{\text {st }}$ century, we approached the practical model employed by the Department of Psychology substantiated on pedagogical terms. Finally, we invite you to think thoroughly about the relevance and challenges of incorporating methodologies into the psychology training that enhance comprehensive development through experiences that can link students with social reality.
\end{abstract} Keywords: learning and service; competencies; project based learning; 21st century skills 


\section{Introducción}

Hoy en día existe una preocupación importante por enriquecer cada vez más los espacios pedagógicos en el contexto universitario y específicamente en la formación profesional de Psicología, uno de los grandes desafíos es la incorporación de experiencias educativas que integren permanentemente el conocimiento con las habilidades prácticas.

El crecimiento de la población de adultos mayores respecto a décadas pasadas, el crecimiento de la población migrante en Chile y la multiculturalidad asociada; la urgencia evidente de atender a temáticas de equidad de género, las problemáticas que surgen a partir de la desigualdad social, la exclusión y la alta prevalencia de trastornos asociados a salud mental en Chile son sólo algunos ejemplos de los desafíos que deberá atender el futuro profesional desde su quehacer, pero estos desafíos van cambiando y sus características dependerán del contexto específico, por tanto resulta altamente relevante que el profesional egresado de Psicología, a lo largo de su formación, desarrolle la capacidad de abordar y generar propuestas desde distintas perspectivas y en diversos contextos y para eso es necesario que cuenten no solamente con conocimientos disciplinares, sino que también potencien habilidades que le permitan adaptarse, aplicar sus conocimientos de distintas maneras según la realidad particular a la que se enfrenten y puedan innovar en el diagnóstico, diseño, intervención y evaluación de las problemáticas emergentes en materia de salud mental, bienestar y desarrollo social y laboral.

En ese contexto es que adquiere relevancia la formación orientada a competencias, la cual implica trabajar tanto el saber de la disciplina en cuanto a contenidos y bases teóricas para la praxis, como también el saber ser, considerando aspectos éticos, morales; que entiendan y se apropien de temáticas como la justicia social, los derechos humanos y la responsabilidad social y que además desarrollen el saber hacer que se traduce en proponer, cambiar y tomar decisiones de 
intervención desde distintas posiciones considerando el contexto cambiante y dinámico y ello sin duda exige un replanteamiento de la docencia, pues resulta fundamental incorporar continuamente en el proceso formativo metodologías activo participativas vinculadas a situaciones reales, en las que el estudiante desarrolle sus conocimientos desde la práctica trabajando con temáticas emergentes y en las que el docente se convierta en un facilitador del aprendizaje poniendo al centro al estudiante como gestor se su propio proceso formativo.

Así, frente a los amplios desafíos de la educación superior y las competencias y habilidades que se espera desarrollar en la formación de profesionales competentes y con un desarrollo integral, luego de la implementación de la formación por competencias en el año 2016, en la Escuela de Psicología de la Universidad Católica Silva Henríquez, se ha trabajado sobre la base del desarrollo de competencias y se han ido incorporando distintas metodologías que acerquen al estudiante al entorno real, a la praxis y a las problemáticas que experimenta hoy la sociedad y en ese contexto, la Metodología Aprendizaje + Servicio (en adelante $A+S$ ) ha sido una herramienta pedagógica que ha permitido avanzar hacia una formación integral, aplicada y valórica pues los estudiantes, bajo esta metodología, se acercan a distintas comunidades para levantar diagnósticos, diseñar e intervenir a través del trabajo directo con socios comunitarios en su propia realidad social, haciendo eco de las necesidades sentidas por la comunidad y vinculándolas con la formación disciplinar y el desarrollo personal de los futuros profesionales.

En la carrera de Psicología de la UCSH, esta metodología si bien se utiliza en distintos cursos de la formación y a nivel transversal en la Universidad, una de las actividades curriculares (en adelante AC) claves en la que se ha demostrado su efectividad para el proceso de enseñanza aprendizaje, es Prácticas Tempranas, que se constituye en el primer curso de aplicación de la carrera, situándose en el nivel 400 del plan de estudios. 
Cabe destacar que una revisión de las mallas curriculares de 10 otras universidades con sede en Santiago y/o regiones de Chile que ofrecen la carrera de Psicología el año 2020, ninguna de ellas ofrece practica temprana, aun cuando al menos dos de ellas otorgan a través de salidas intermedias certificaciones como bachillerato en comportamiento humano, minor en Psicología o evaluaciones de perfil intermedio, siendo esto una evidencia de la mantención de un modelo educativo centrado en la teoría y con un tardío acercamiento al mundo laboral en que se desempeñaran los futuros psicólogos y psicólogas ${ }^{1}$.

A continuación se presentan los desafíos de la formación profesional desde el análisis de Elmore (2010) situado en educación superior, Tobón (2010 en Medina 2011) y UNESCO (1998 en Ministerio de Educación 2007); luego se aborda el cómo la Metodología A+S desde el modelo de CLAYSS (2016) y el Ministerio de Educación (2007) permitiría responder a dichos desafíos, para luego presentar el modelo utilizado en el curso Práctica Temprana de Psicología UCSH que considera tanto la metodología A+S de CLAYSS como el Aprendizaje basado en Proyectos (en adelante ABP) de Heydrick, Rojas y Hernández (2010). Finalmente se realiza un análisis y se plantean diversas reflexiones respecto al aporte pedagógico de la metodología práctica en relación a las habilidades y competencias necesarias de desarrollar en el siglo XXI desde el análisis de AMA (2010 en Hodge \& Lear, 2011) y APEC (2008 en Scott, 2015) y el Ministerio de Educación (2007).

\footnotetext{
${ }^{1}$ Se revisaron las mallas curriculares de las siguientes Universidades Acreditadas de la Región Metropolitana y V región de Chile, a saber: Santo tomas, San Sebastián (salida intermedia como Bachiller en comportamiento humano, Nacional Andrés Bello, Pontificia Universidad Católica de Chile, De Chile, Pontificia Universidad Católica de Valparaíso, de Valparaíso, de los Andes, Diego Portales y Alberto Hurtado
} 


\section{Antecedentes teóricos y conceptuales: Formación por competencias y \\ Aprendizaje + Servicio}

En docencia universitaria se ha ido observando una evolución paulatina respecto a las metodologías y didácticas que buscan la integración del desarrollo de habilidades, destrezas y conocimientos para el quehacer profesional. Hoy es posible evidenciar un crecimiento importante en los programas de perfeccionamiento docente y en la utilización de metodologías en las que se pone al estudiante al centro del proceso de enseñanza aprendizaje y en las que adquiere cada vez más relevancia la interacción entre los contenidos, el docente y el estudiante, es decir el núcleo pedagógico (Elmore, 2010). Es esta interacción, en la participación activa de los involucrados y en lo sucede en el proceso lo que determina el real aprendizaje del estudiante, debido a que necesariamente el aprendizaje requiere una experiencia directa y en primera persona para asegurar la implicación, motivación y trascendencia de los contenidos y habilidades que se busca potenciar en el futuro profesional. Esta afirmación aplica, a juicio de las autoras, a todos los niveles de la educación formal incluyendo la formación universitaria.

"El cambio en los conocimientos y las habilidades que los profesores incorporan en sus prácticas, el tipo de contenido al cual tienen acceso los alumnos y el rol que ellos juegan en su propio aprendizaje, son los elementos que determinan lo que aprenderán los estudiantes y lo que serán capaces de hacer" (Elmore, 2010, p.21). En ello se sustenta la importancia de la aplicación práctica, el acompañamiento y reflexión que se genera en el proceso de aprendizaje y si a ello le sumamos lo fundamental que resulta el compromiso social y la preocupación que se espera tengan los profesionales de Psicología por el bien común, el compromiso solidario y transformador que sobre todo la Universidad Católica Silva Henríquez promueve, resulta necesario incorporar 
metodologías que no sólo sean participativas, sino que también vinculen al estudiante con la realidad social en la que están insertos.

Así, en atención a los desafíos educativos de la educación superior en la actualidad y a las demandas del contexto laboral y social en el que se insertan los egresados de Psicología, la formación en Psicología de la Universidad Católica Silva Henríquez en la actualidad ha ido actualizando sus metodologías, estrategias didácticas y procesos de práctica avanzando hacia un modelo orientado a competencias. Hoy uno de los focos relevantes es atender a lo que sucede directamente en el proceso de enseñanza aprendizaje de las actividades curriculares para el desarrollo efectivo de las competencias declaradas en el perfil de egreso, pues si se espera que el egresado se posicione teóricamente para el diagnóstico, diseño, intervención, evaluación e investigación con una preocupación permanente por las problemáticas emergentes desde una perspectiva psicosocial es esperable que el Plan de estudios en su conjunto y su operacionalización permita alcanzar dicho propósito formativo a través de la adquisición de competencias no sólo procedimentales y disciplinares, sino que también transversales.

Existen tres ejes competenciales en los procesos de formación de los estudiantes: el laboralempresarial, la integración sociocultural y la autorrealización; esto es, que las personas puedan formarse para ser eficaces, para ser solidarias con los demás y para gestionar su propio proyecto ético de vida (...) alguien es competente cuando puede integrarse en una tarea con los demás, es decir, aprender a ser competente es formarse en la concepción personal, cultural y socio-laboral. (Tobón, 2010 en Medina, 2010). Para el autor las competencias contemplan el saber ser, saber conocer y saber hacer, las divide en competencias básicas, genéricas y específicas y propone la articulación entre el proceso de aprendizaje siguiendo el principio dialógico del pensamiento complejo, la metacognición y la actuación idónea. Por tanto, el modelo por competencias implica 
pensar de manera integrada y sistémica el proceso de enseñanza aprendizaje en la que el estudiante desarrolla habilidades integrales que le permitirán aplicar conocimientos, pero también, autoregularse, pensarse y considerar los aspectos éticos de su intervención.

La formación de profesionales competentes y comprometidos con el desarrollo social constituye hoy en día una misión esencial de la educación superior (UNESCO, 1998 en González, Herrera \& Zurita, 2008) y esto se refleja sin duda en la formación de Psicólogos y Psicólogas ya que es indispensable el desarrollo de competencias que permitan analizar e intervenir en su entorno considerando la cultura, valores e historia del contexto entendiendo las problemáticas actuales y la interacción entre lo psicológico y social de los fenómenos, por tanto la formación bajo esta línea y la aplicación de metodologías que integren el aprendizaje y el servicio se vinculan de manera directa con las necesidades de formación en la disciplina, pues a través de la aplicación en contextos comunitarios reales, es posible el desarrollo de todos los factores competenciales necesarios para la praxis profesional.

La formación de la solidaridad es un camino necesario para desarrollar la educación integral y de calidad que nuestra sociedad requiere. La comisión internacional sobre la Educación para el siglo XXI, en su informe a la UNESCO identifica cuatro pilares sobre los que debe asentarse la educación: Aprender a conocer, aprender a hacer, aprender a ser y aprender a vivir juntos" (Ministerio de Educación, 2007). La Metodología Aprendizaje y Servicio abre sin dudas este camino, pues permite la interacción e integración de estos 4 pilares en su aprender sirviendo, conviviendo y comprendiendo contextos reales de intervención durante la formación de pregrado. Desde la perspectiva de las habilidades para el siglo XXI, a saber, la creatividad, la resolución de problemas, el pensamiento crítico, la colaboración también llamadas las 4 C propio de la educación en cuatro dimensiones, se articulan armónicamente con la metodología $\mathrm{A}+\mathrm{S}$ ya que fortalecen una 
formación integral, adaptativa, basada en las necesidades locales que promueve la elección consciente de opciones y, sobre todo, no limita la formación a la transmisión de conocimiento como única meta.

En ese contexto es que la Metodología $\mathrm{A}+\mathrm{S}$ ha ido cobrando relevancia en el tiempo y se ha ido posicionando como una estrategia pedagógica al servicio de la formación de Psicólogos y Psicólogas en la carrera Psicología de la UCSH siendo considerada como la metodología oficial de las Prácticas iniciales de la carrera. Pero realizar una intervención en contexto real no necesariamente se constituye en una experiencia A+S. Existen ciertas características propias de la metodología que resulta importante revisar para determinar su aplicación efectiva como herramienta pedagógica que permite el desarrollo de competencias.

Tapia (2006) considera el A+S como una filosofía educativa, que subraya el valor de las actividades solidarias al servicio de la comunidad y desarrolla su potencial educativo, conectándolas con el aprendizaje formal Tapia, 2006 en Jouannet, Salas, \& Contreras, 2013). Por su parte, CLAYSS (2016), el Centro Latinoamericano de Aprendizaje y Servicio solidario, define el $\mathrm{A}+\mathrm{S}$ como una propuesta pedagógica innovadora que permite contribuir tanto en la mejora de la calidad de vida de las comunidades como en la calidad de la oferta educativa formal y no formal a través de la aplicación de conocimientos al servicio de las necesidades.

Es en esta acción en la que los estudiantes aprenden nuevos conocimientos, desarrollan competencias para la vida, el trabajo y para la participación ciudadana pues esto sólo resulta posible en el haciendo. Aprendemos a hacer el trabajo haciendo el trabajo, no diciéndole a otras personas que lo hagan, no por haberlo hecho alguna vez en el pasado, y no contratando a expertos que pueden actuar como sustitutos de nuestro conocimiento acerca de cómo hacerlo (Elmore, 2010). Pero si a este hacer se le suma la entrega de un servicio social o comunitario, se logra 
generar un potente complemento entre el aprender haciendo y el aprender siendo y contribuyendo a la sociedad desde el quehacer profesional. Es en esta experiencia donde el servir permite la adquisición de herramientas y valores que van más allá de una revisión bibliográfica y de contenidos disciplinares, pero para CLAYSS (2016) existen tres rasgos fundamentales para que la metodología $\mathrm{A}+\mathrm{S}$ se traduzca en aprendizaje activo:

1. Servicio solidario para atender a necesidades reales y sentidas por la comunidad.

2. Protagonismo de los estudiantes

3. Planificación integrada con los contenidos curriculares de aprendizaje e investigación.

Se espera entonces que en la actividad curricular en la que se incorpora la metodología se logre un equilibrio entre la aplicación autónoma de contenidos disciplinares y la entrega de un servicio a la comunidad lo cual otorga una serie de beneficios a nivel pedagógico pues se integra la teórica, la práctica, la formación ciudadana y la responsabilidad social además de una serie de competencias transversales asociadas al desarrollo profesional.

Jouannet, Salas y Contreras (2013) concluyen en su estudio modelo de implementación de aprendizaje servicio $(\mathrm{A}+\mathrm{S})$ en la Pontificia Universidad Católica de Chile que el modelo permite en los estudiantes el desarrollo de habilidades, actitudes y valores como el compromiso social, el trabajo en equipo y la resolución de problemas posicionando la metodología como una herramienta eficaz para la formación de profesionales competentes y comprometidos con la sociedad. De esta forma la acción de contribuir al desarrollo, educación, transformación y bienestar de una comunidad permite un desarrollo integral fortaleciendo valores y la responsabilidad ciudadana requerida sino indispensable para los futuros profesionales.

Por su parte, el Ministerio de Educación (2007) señala que A+S además permite a los estudiantes descubrir, aplicar y profundizar los conceptos disciplinarios en la relación que 
establecen con situaciones reales y problemas concretos además de facilitar un mayor desarrollo en la comunidad local, considerándose así una herramienta pedagógica eficaz que posibilita el actualizar y verificar lo estudiado, dar sentido a lo que ya se sabe, motivar a investigar lo que no se sabe y desplegar la creatividad e iniciativa de los estudiantes.

De esta forma el estudiante adquiere conocimientos aplicados y una formación social responsable a través de experiencias protagónicas en la que pone al servicio de una comunidad sus saberes y se sitúa al mismo tiempo en el centro del proceso de enseñanza aprendizaje ya que desde la perspectiva constructivista el docente guía y orienta la acción como un mediador y/o facilitador y el estudiante se apropia y gestiona su acción en el mundo social potenciando la autorregulación, la implicación y la reflexión sobre el propio quehacer. Así es que A+S presenta diversos beneficios académicos.

El Ministerio de Educación (2007), señala distintas motivaciones de carácter pedagógico para incorporar la metodología, entre las cuales se encuentra la profundización del aprendizaje, el desarrollo de competencias, la capacidad para resolver problemas, el lograr aprendizajes integrados y el que promueve la integración con otros para la construcción de conocimiento, entre otros. Pero además para lograr un aprendizaje significativo es necesario incorporar en la praxis la reflexión de la experiencia. El pensamiento reflexivo, es clave para lograr que las experiencias educativas se conviertan en conocimiento y que éstas conduzcan a una acción socialmente comprometida, pero es importante que las tareas de reflexión estén conectadas a los objetivos de aprendizaje para impulsar el pensamiento y análisis profundo de uno en relación con la sociedad (Opazo, 2015).

La estrategia de reflexión en $\mathrm{A}+\mathrm{S}$ es entendida como el diseño de espacios en el curso antes, durante y después de la experiencia de servicio, que apuntan a que los estudiantes logren 
una comprensión profunda de los aspectos históricos, sociológicos, culturales, económicos y políticos que están detrás de las necesidades de la comunidad (Cooper, Cripps y Reisman, 2013 en Jouannet, Salas \& Contreras, 2013). Así también lo señala CLAYSS (2016) cuando releva el interrogarse sobre la relevancia social de los aprendizajes adquiridos en el proceso y sobre la pertinencia de esos conocimientos en función de las necesidades de la comunidad. El rol de la reflexión en A+S, siguiendo Hansen (2012) y Eyler (2009) citados por Jouannet, Salas, y Contreras (2013) es central y debe ser una reflexión estructurada para que sea efectiva.

La organización de la actividad curricular que contemple la aplicación de la metodología $\mathrm{A}+\mathrm{S}$ entonces debe generar necesariamente un espacio de reflexión mediado por los docentes y debe organizar una secuencia de trabajo metacognitiva que aborde un espectro amplio de preguntas y temas con el fin de permitir la revisión sistemática, consciente y personal entre pares y con el mediador no sólo sobre el qué se aprende y cómo se aprende desde el propio proceso de aprendizaje sino que sobre todo cómo esto se vincula con la realidad social concreta. Fuentes y Rosario (2013) proponen que, con el fin de potenciar la autorregulación, los modelos pedagógicos sistemáticamente deben realizar un ciclo en espiral de planificación, ejecución y evaluación de la acción en forma constante evitando los momentos aislados de reflexión ya que estos por sí solos y de manera aislada no impactan en el proceso cognitivo. De esta forma resulta fundamental comprender cómo se aplica la metodología en la praxis considerando cada uno de los elementos pedagógicos en equilibrio.

Ahora bien ¿Cómo se operacionaliza esto en la práctica? El Centro Latinoamericano de aprendizaje y servicio solidario ha puesto énfasis en el proceso reflexivo y en la aplicabilidad de los proyectos basados en $\mathrm{A}+\mathrm{S}$, proponiendo un itinerario de Proyecto basándose en los planteamientos de Tapia (2006) en CLAYSS (2016): 
1. Etapa 1 "Motivación": Esta etapa considera un proceso motivacional y de conocimiento y comprensión del concepto $\mathrm{A}+\mathrm{S}$ y de la relevancia del protagonismo del estudiante

2. Etapa 2 "Diagnóstico": Como segundo paso se espera la identificación de necesidades detectadas en conjunto con la comunidad y el análisis de la factibilidad de la respuesta desde la institución educativa

3. Etapa 3 "Diseño y planificación": En esta fase se deben identificar los objetivos del servicio y del aprendizaje, identificar a los destinatarios, las actividades, los contenidos y actividades de aprendizaje, los tiempos asociados, lugares, recursos, responsables y la reflexión y evaluación del diseño del proyecto

4. Etapa 4 "Ejecución": Como cuarto paso se espera que los estudiantes establezcan alianzas, lleguen a acuerdos e implementen el proyecto registrando la acción, evaluando el proceso, los logros y realicen los ajustes necesarios.

5. Etapa 5 "Cierre y multiplicación": Esta etapa se constituye en la evaluación y sistematización final, reconocimiento de los protagonistas y multiplicación o continuidad del proyecto en el tiempo.

6. Proceso Transversal "Reflexión": Se trata del registro, sistematización, comunicación y evaluación permanente en el proyecto.

(Tapia, 2006 en CLAYSS, 2016)

Estas etapas se consideran fundamentales para lograr los propósitos educativos y los resultados de aprendizaje esperados en actividades curriculares de diagnóstico e intervención psicosocial en Psicología pues permite que los y las estudiantes planteen interrogantes, investiguen, identifiquen problemáticas, posibles respuestas e intervengan en una realidad social desde la aplicación teórica. Así estas estas etapas se consideraron como base para la formulación 
del modelo metodológico general utilizado en Prácticas Tempranas de Psicología de la Universidad Católica Silva Henríquez donde el producto esperado es un proyecto de intervención psicosocial aplicado en comunidades.

\section{Caracterización de Prácticas iniciales en el contexto de formación por competencias de la carrera de Psicología de UCSH}

La Práctica temprana en la carrera Psicología de la UCSH se ubica en el eje de formación práctica del plan de estudios y busca que los estudiantes diseñen e implementen un proyecto de intervención psicosocial que sea pertinente y relevante para una determinada comunidad. Ello con el fin de desarrollar la responsabilidad social y ciertas habilidades iniciales de diagnóstico, diseño e intervención vinculadas con competencias específicas de la carrera de acuerdo al perfil de egreso como lo son:

- Diagnosticar fenómenos y procesos psicológicos en personas, grupos y organizaciones considerando el contexto social y los procedimientos e instrumentos teórico- prácticos de diversos campos profesionales de la psicología.

- Expresar los resultados del proceso diagnóstico a través de un lenguaje técnico propio de la psicología en forma oral y escrita

- Diseñar iniciativas pertinentes y relevantes en diversos campos profesionales de la psicología destinadas a personas, grupos y organizaciones.

- Emitir un juicio fundado sobre la calidad y aporte de procesos de intervención desarrollados en personas, grupos y organizaciones en base a criterios teórico-prácticos pertinentes y relevantes.

Así, basándose en el propósito formativo de la AC, en los planteamientos del modelo A+S de CLAYSS y en los fundamentos de la formación orientada a competencias, la Escuela de 
Psicología de la UCSH ha elaborado un modelo específico de intervención para esta Actividad curricular incorporando el trabajo grupal en terreno con socios comunitarios bajo la supervisión y acompañamiento de docentes expertos en intervención psicosocial.

En el proceso se espera que a través del trabajo en grupos guiados por el o la docente comprendan en primera instancia los fundamentos, la lógica y los procesos involucrados en la elaboración de proyectos psicosociales, para luego insertarse en una comunidad durante 6 horas semanales por 4 meses. Durante este tiempo, inician su trabajo con la comprensión de las necesidades del socio comunitario en su propio contexto, analizan y reflexionan sobre las problemáticas y diseñan con fundamentos teóricos una intervención contextualizada que luego deben presentar en una feria abierta a la comunidad local y universitaria. Todo ello acompañado de clases presenciales de 4 horas a la semana en las que se van revisando aspectos teóricos, reflexiones personales y grupales y avances de los distintos proyectos.

\section{Modelo de intervención psicosocial Psicología UCSH basado en A+S con aplicación de metodología de Aprendizaje basado en proyectos}

El modelo de intervención propuesto por la carrera desde el año 2017 (año de su primera implementación) contempla distintas etapas y se sustenta en los fundamentos de A+S y del Aprendizaje basado en proyectos (en adelante $\mathrm{ABP}$ ).

El ABP "es un modelo de aprendizaje con el cual los estudiantes trabajan de manera activa, planean, implementan y evalúan proyectos que tienen aplicación en el mundo real más allá del aula de clase” (Blank, 1997; Harwell, 1997; Martí, 2010 en Martí, Heydrick, Rojas y Hernández, 2010, p.13). Una de los elementos centrales de esta metodología activa como estrategia didáctica es abordar problemas complejos sin soluciones obvias dentro del mundo real a través de la búsqueda activa de soluciones por parte de los educandos. Así durante el semestre los estudiantes 
deben diseñar un proyecto mediante la realización de distintas tareas diagnósticas de diseño, elaboración, evaluación, presentación y socialización de un producto innovador de impacto social para una determinada comunidad incorporando como estrategia transversal la reflexión para conectar la teoría y los conocimientos con la práctica y repensar permanentemente el quehacer y la responsabilidad profesional.

Martí, Heydrick, Rojas y Hernández (2010) proponen que la ABP permite entre otros objetivos, mejorar la habilidad para resolver problemas y desarrollar tareas complejas, mejorar la capacidad de trabajar en equipo, desarrollar las capacidades mentales de orden superior, promover una mayor responsabilidad por el aprendizaje propio. Concluyen que en la formación de pre y post grado esta metodología permite al mismo tiempo potenciar el trabajo y dejar de lado la enseñanza mecánica y memorística para enfocarse en un trabajo más retador y complejo utilizando un enfoque interdisciplinario y el trabajo cooperativo de los participantes. Por este motivo es que los productos esperados en la AC son diversos en su forma, contenido y complejidad incluyendo entre ellos informes diagnósticos fundamentados desde la teoría, devoluciones a socios comunitarios, informes de diseño y planificación del proyecto, bitácoras personales de aprendizaje y socialización del proyecto o intervención ejecutada a través de ferias de exposición abiertas a la comunidad.

En consecuencia, considerando los aportes de ambos modelos metodológicos ( $\mathrm{A}+\mathrm{S}$ y $\mathrm{ABP})$ y los objetivos pedagógicos de la actividad curricular presentados en el apartado de caracterización, las etapas que constituyen el modelo de intervención psicosocial en el curso son los siguientes:

1. Etapa 1: Iniciación del proceso e incorporación a la institución: En esta fase el estudiante conoce sobre los fundamentos del Aprendizaje y Servicio, la elaboración de proyectos, 
los propósitos de la actividad curricular y su vínculo con el perfil de egreso y se prepara para su ingreso al centro de Práctica (Primer acercamiento al socio comunitario)

2. Etapa 2: Diagnóstico: En esta etapa, el grupo de estudiantes ya insertos en el contexto de práctica, a través de diagnósticos participativos identifican necesidades, problemas y desafíos que presenta la comunidad finalizando con un árbol de problemas a presentar a la comunidad con un análisis profundo considerando elementos teóricos aplicables a la problemática.

3. Etapa 3: Diseño y Planificación del proyecto: Luego de identificar la problemática principal, urgente y sentida por la comunidad se plantea en conjunto con el docente, los objetivos del proyecto y su fundamentación teórica para luego diseñar la intervención con actividades específicas, responsables, destinatarios, cronograma y recursos asociados. Es en esta fase y en la anterior donde juega un papel fundamental el vínculo entre el saber y el saber hacer.

4. Etapa 4: Evaluación de la intervención: En base a criterios teórico prácticos, se evalúa la aplicabilidad y beneficios del proyecto y se abre el espacio a la reformulación si ello fuese necesario. En este punto juega un papel fundamental el saber ser, la reflexión en torno al propio saber y a la responsabilidad respecto a los resultados esperados.

5. Etapa 5: Ejecución: A través de la autogestión, organización, coordinación con los socios comunitarios y aplicación del proyecto se espera favorecer el aprendizaje autoregulado y significativo. Es en esta fase donde adquiere mayor relevancia la aplicación del conocimiento.

6. Etapa 6: Cierre y socialización: Esta se constituye en la etapa final de intervención en la que los estudiantes integran tanto el saber, el saber ser y el saber hacer a través de la 
reflexión de los resultados, el aprendizaje adquirido y la contribución al desarrollo y aprendizaje de la comunidad. A través de la presentación del proyecto de intervención psicosocial en una feria abierta a la comunidad, los y las estudiantes presentan y fundamentan su intervención abriendo espacios conjuntos de reflexión y diálogo con docentes, estudiantes y sociocomunitarios respecto al proyecto.

7. Proceso Transversal: Reflexión: En cada una de las etapas del proyecto y en la cotidianidad de la práctica, los estudiantes trabajan en torno a una bitácora o diario de aprendizaje; en cada visita semanal a la institución del socio comunitario, cada estudiante, registra las acciones, tareas, aciertos, aspectos a mejorar, emociones y reacciones a través de una reflexión crítica y analítica de la experiencia facilitando una conexión entre el proyecto, sus aprendizajes y desarrollo personal, social y profesional. A ello se suma una autoevaluación intermedia y final y una coevaluación que permite la reflexión conjunta de los aportes de cada uno de los miembros.

Respecto a los resultados, a la fecha se han desarrollado tres procesos de implementación bajo el modelo para las cohortes 2017, 2018 y 2019, donde un total de 329 estudiantes de la carrera se han incorporado en distintos espacios comunitarios de práctica alcanzando el logro de los indicadores de aprendizaje específicos de la AC.

Tabla $\mathrm{N}^{\circ} 1$ :

Estudiantes y proyectos por año escuela de Psicología UCSH (2017-2019)

\begin{tabular}{ccc}
\hline $\begin{array}{c}\text { Año de realización de } \\
\text { práctica temprana }\end{array}$ & $\begin{array}{c}\text { Número de estudiantes } \\
\text { (Total 329) }\end{array}$ & $\begin{array}{c}\text { Número de Proyectos } \\
\text { (Total 86) }\end{array}$ \\
\hline $\mathbf{2 0 1 7}$ & 107 & 28 \\
$\mathbf{2 0 1 8}$ & 117 & 30 \\
$\mathbf{2 0 1 9}$ & 105 & 28 \\
\hline
\end{tabular}

Fuente: Elaboración propia en base a datos de Escuela Psicología UCSH[ML1] 
Respecto al tipo de usuario o población objetivo, se han seleccionado socios comunitarios que cuentan con características que permitan al estudiante desarrollar acciones socialmente comprometidas. Así, la línea que han seguido los centros de Práctica y el tipo de intervención generalmente son los siguientes:

Tabla N²:

Tipos de socio comunitario y ámbito de intervención

Tipos de Socios

\section{Comunitarios}

\section{Ámbitos de intervención}

Habilidades socioemocionales - clima - sexualidad y

afectividad - autocuidado - comunidades pedagógicas inclusión - convivencia escolar- proyecto de vida

Residencias de adulto Mayor

Fortalecimiento de habilidades - Recreación - Duelo Integración y comunicación grupal - Bienestar Psicológico

Comunidades Migrantes

Intercambio cultural - Derechos - Acceso a información

Centros de día para personas

Habilidades sociales - Espacios recreativos y culturales en situación de calle Relaciones interpersonales

Centros de formación de Herramientas pedagógicas - Vocación y Proyecto de vidaadultos Clima y convivencia
Inclusión - Salud Mental - Gestión de voluntariados

Fuente: Elaboración Propia en base a datos Escuela Psicología

En su inserción en este tipo de centro de práctica y de acuerdo a lo que se ha evidenciado en la experiencia práctica docente, se ha logrado que los estudiantes desarrollen una sensibilidad especial por los sectores más vulnerables de la sociedad adquiriendo a su vez un compromiso importante por la educación, el desarrollo social, la inclusión, los derechos ciudadanos y el 
bienestar de las personas, lo cual se ha evidenciado tanto en los resultados de aprendizaje analizados bajo rúbricas que miden los indicadores de aprendizaje específicos de la AC como también en sus bitácoras, reflexiones y presentaciones en las ferias abiertas a la comunidad.

Así, a través del trabajo en terreno tres generaciones de estudiantes han podido conocer desde su propia experiencia las realidades y problemáticas de distintos sectores de la sociedad aplicando y vinculando los conocimientos disciplinares adquiridos en la formación inicial.

Además, en el trabajo conjunto entre estudiantes y docentes se ha incentivado permanentemente el trabajar en torno a la resignificación de la experiencia a través del diálogo y el análisis de la experiencia en relación a saberes teóricos ya que el papel que juega la metacognición y la reflexión de la praxis se han considerado aspectos fundamentales en el proceso de aprendizaje y en el logro de los objetivos pedagógicos de la AC.

\section{Reflexiones desde la didáctica sobre $\mathrm{A}+\mathrm{S}$ y el aprendizaje basado en proyectos como} metodología de enseñanza aprendizaje en la formación de pregrado de Psicólogos.

El Consejo Nacional de Educación siguiendo a Fadel, Bialik \& Trilling (2017) en Educación en cuatro dimensiones plantea la necesidad de poner énfasis tanto en el tipo de proceso educativo en que se basa la formación profesional como en el mundo laboral futuro en que se insertarán los egresados con el fin de aumentar su empleabilidad. Se debe tener en cuenta, el tipo de trabajo que ejecutarán los educandos al integrarse al mundo laboral en el siglo XXI al momento de implementar el currículo de pregrado sin perder de vista los contenidos clásicos y propios de la disciplina. Este aspecto se relaciona directamente con el perfil de egreso esperado en la formación de pregrado en psicología.

Si bien a nivel internacional no existe un modelo ni una forma univoca de nombrarlas que determine las habilidades, competencias o conocimientos necesarios para el siglo XXI, si existen 
ciertos grados de acuerdo entre diversos organismos nacionales e internacionales como el Ministerio de Educación (2007), APEC (2008 en Scott, 2015), AMA 2010 en Hodge \&Lear, 2011), entre otros como se muestra en la siguiente tabla donde se muestran agrupadas y se busca graficar sus elementos comunes:

Tabla N³:

Elementos claves de la Educación para el siglo XXI

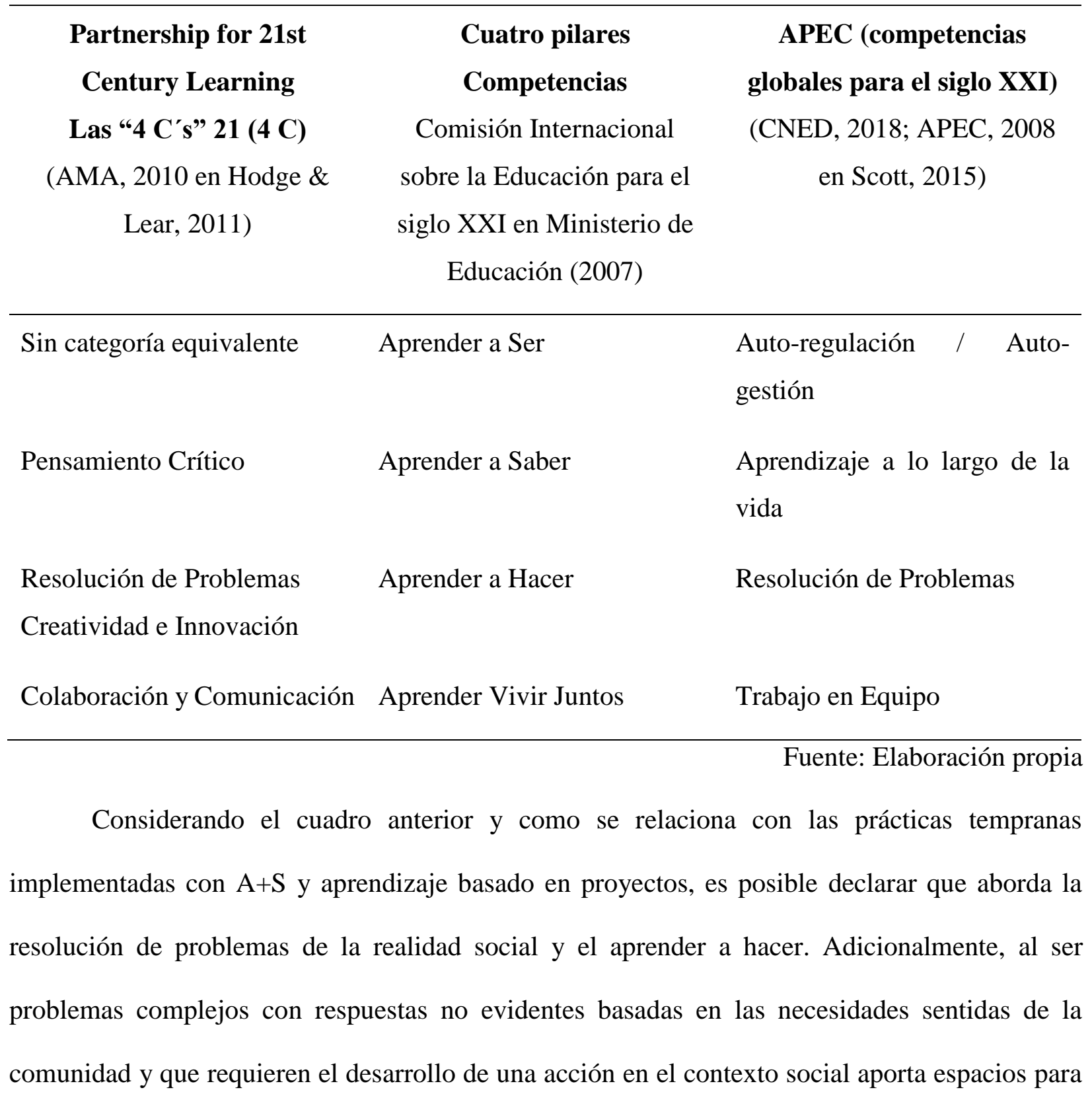


el desarrollo de la creatividad e innovación. Al ser un aprendizaje que se realiza en equipos, es decir, de tipo social potencia además el desarrollo de la colaboración y comunicación aportando al trabajo en equipo en términos de la APEC o el aprender a vivir juntos en la propuesta de UNESCO de los 4 pilares. A su vez, el trabajo de terreno, que se desarrolla en el campo social en las instalaciones y territorio del socio comunitario y por tanto fuera del aula y del contexto universitario, implica una relación entre los pares que pone al centro las habilidades interpersonales como la colaboración y la comunicación.

En la tabla $\mathrm{N}^{\circ} 4$ se abordan las operaciones mentales que el modelo por etapas de $\mathrm{A}+\mathrm{S}$ aplicado en la escuela de Psicología a las practicas tempranas busca desarrollar. La incorporación del aprendizaje basado en proyectos en las practicas tempranas, busca potenciar las operaciones mentales superiores, entendidas en forma genérica como un conjunto de acciones interiorizadas y organizadas por las que elaboramos la información que proviene de fuentes internas y externas. Debido a que no se puede desarrollar el pensamiento sin un contenido, y que no se puede aprender un contenido sin un proceso de pensamiento al trabajar en ABP se activan el análisis, síntesis, la recolección de información, el pensamiento crítico, el razonamiento inductivo y deductivo.

Al participar en la realidad social con el fin de proponer un proyecto se activa la unión indivisible y bidireccional entre el proceso cognitivo y el contenido, que es la clave para abordar estos desafíos educativos en el aula universitaria.

La metodología de proyecto utilizada en Prácticas Tempranas de Psicología UCSH bajo los fundamentos del modelo A+S, supone el desarrollo de un producto, presentación y/o actuación en una feria de prácticas tempranas ante sus pares y docentes de la carrera, permitiendo potenciar tanto las habilidades comunicativas escritas y orales de los psicólogos y psicólogas en formación en torno a un proceso ejecutado en la realidad social. Además, la estructura misma de la actividad 
curricular con trabajo de aula y de terreno el que se registra en un diario de campo, el contacto con el docente supervisor de la UCSH quien actúa tanto como docente de aula y como mediador en grupos pequeños de estudiantes, busca potenciar la metacognición y por tanto la autorregulación. Ya que esta metodología promueve la autogestión y el desarrollo de un rol activo sobre el propio aprendizaje.

Tabla $\mathrm{N}^{\circ} 4$ :

Análisis de la aplicación del modelo de intervención en base a las competencias y operaciones mentales que se desarrollan a través de la aplicación de la metodología

\begin{tabular}{|c|c|c|}
\hline $\begin{array}{l}\text { Etapas de aplicación A+S } \\
\text { de la Escuela de Psicología }\end{array}$ & $\begin{array}{c}\text { Pilares de la formación por } \\
\text { competencias en base a }\end{array}$ & $\begin{array}{c}\text { Las } 4 \text { C AMA y las } \\
\text { Competencias globales para }\end{array}$ \\
\hline \multirow[t]{3}{*}{ UCSH basado en CLAYSS } & $\begin{array}{c}\text { Tobón (2010) en Medina } \\
\text { (2010) y la Comisión }\end{array}$ & $\begin{array}{c}\text { el siglo XXI APEC } \\
\text { (AMA, } 2010 \text { en Hodge \& }\end{array}$ \\
\hline & Internacional & Lear, 2011; CNED, 2018; \\
\hline & $\begin{array}{l}\text { sobre la Educación para el } \\
\text { siglo XXI en Ministerio de }\end{array}$ & APEC, 2008 en Scott, 2015) \\
\hline
\end{tabular}

Educación (2007)

\begin{tabular}{|c|c|c|}
\hline $\begin{array}{l}\text { 1: Iniciación del proceso e } \\
\text { incorporación a la institución }\end{array}$ & Saber ser, & $\begin{array}{l}\text { Colaboración } \\
\text { Aprendizaje a lo largo de la } \\
\text { vida }\end{array}$ \\
\hline 2: Diagnóstico & Saber y Saber hacer & $\begin{array}{l}\text { Resolución de Problemas } \\
\text { Pensamiento Crítico } \\
\text { Colaboración }\end{array}$ \\
\hline $\begin{array}{l}\text { 3: Diseño y Planificación del } \\
\text { proyecto }\end{array}$ & Saber y Saber hacer & $\begin{array}{l}\text { Resolución de Problemas } \\
\text { Creatividad Innovación }\end{array}$ \\
\hline $\begin{array}{l}\text { 4: Evaluación de la } \\
\text { intervención }\end{array}$ & Saber, Saber ser & $\begin{array}{l}\text { Pensamiento Crítico } \\
\text { Colaboración } \\
\text { Aprendizaje a lo largo de la } \\
\text { vida }\end{array}$ \\
\hline
\end{tabular}




$\begin{array}{lll}\text { 5: Ejecución } & \text { Saber hacer } & \begin{array}{l}\text { Resolución de problemas } \\ \text { Autorregulación }\end{array} \\ \text { 6: Cierre y socialización } & \text { Saber, Saber ser y Saber } & \begin{array}{l}\text { Comunicación } \\ \text { hacer }\end{array} \\ & & \begin{array}{l}\text { Aprendizaje a lo largo de la } \\ \text { vida }\end{array} \\ \text { Proceso Transversal: } & \text { Saber ser } & \text { Metacognición y } \\ \text { Reflexión } & & \text { autorregulación }\end{array}$

Fuente: Elaboración propia

Considerando estos planteamientos y los beneficios de la aplicación de la metodología A+S en el desarrollo de competencias y habilidades del siglo XXI que requerirán los egresados de la carrera, la Escuela de Psicología de la UCSH ha ido apropiándose cada vez más de la metodología como parte importante de la operacionalización del Plan de estudios, así, en la actualidad, la Práctica Temprana está declarada como una actividad curricular con Metodología de proyectos $\mathrm{A}+\mathrm{S}$ que cuenta con distintos espacios de recogida de datos en aula para su sistematización, con un manual docente y además ha ido fortaleciendo permanentemente su modelo de aplicación en el tiempo a través de la evaluación teórica de su impacto en el proceso de enseñanza aprendizaje.

\section{Conclusiones y reflexiones finales}

El psicólogo(a), por la naturaleza del rol y de su quehacer, realiza un amplio rango de tareas en diversos tipos de ambientes laborales sin embargo todos tienen en común ser tareas no rutinarias y personales difíciles de automatizar y de externalizar donde predominan habilidades de tipo interpersonales y analíticas en contextos de alta complejidad y de desafíos no predecibles ni previamente experimentados por las generaciones de profesionales que los forman. Así el contacto con la realidad social en forma temprana durante el proceso formativo a través de las prácticas tempranas constituye sin duda un valor agregado al proceso educativo, siendo la UCSH 
una de las pocas universidades ofrece en el contexto de la formación de pregrado de Psicólogos y Psicólogas en Chile.

Se coincide con los distintos autores revisados como CNED (2018), Tobón (2010) en Medina (2010), AMA (2010 en Hodge \& Lear, 2011) y la Comisión Internacional sobre la Educación para el siglo XXI en Ministerio de Educación (2007) que las tareas que deberán enfrentar los egresados a lo largo de su vida laboral implicarán además de la relación cotidiana y activa con otros profesionales y técnicos de múltiples disciplinas pues ninguna de las ciencias y disciplinas en la actualidad resuelve por sí misma los problemas y desafíos emergentes. Así las habilidades de colaboración, resolución de problemas, pensamiento crítico y comunicación serán centrales para el desarrollo profesional en el futuro y por tano se deben potenciar específicamente a través del currículo.

En base a lo anterior, la Educación en todos los niveles educativos debe abandonar el predominio en el contenido y migrar a una mirada de competencias favoreciendo la integración del conocimiento sobre la memorización y el conocimiento enciclopédico. El proceso formativo debe hacerse cargo de favorecer la adaptación al contexto personal, cívico y no solo el mundo laboral futuro por cierto desconocido para los propios formadores.

Necesariamente las instituciones formadoras de pregrado en Educación Superior, deben hacerse cargo de potenciar la autonomía y la capacidad de transferir de un contexto a otros elementos centrales para el desempeño en un mundo de alta complejidad y la metodología $\mathrm{A}+\mathrm{S}$ incorporando el ABP han logrado impactar en estos ámbitos desarrollando habilidades que permitirán al egresado enfrentar los desafíos de la sociedad y sus múltiples comunidades. El modelo de A+S aplicado por la carrera de Psicología de la UCSH a partir del aprendizaje implementado por medio aprendizaje basado en proyectos y el desarrollo de habilidades de 
aplicación para la innovación social, potencia el desarrollo de habilidades centrales para el futuro desarrollo profesional como son la metacognición, el trabajo colaborativo y en general las operaciones mentales de orden superior.

Entre los desafíos y proyecciones del modelo actual se encuentra la necesidad de reconocer, siguiendo a Jouannet, Salas \& Contreras (2013) en Calidad de la Educación, de manera efectiva, las horas de trabajo docente que implica la incorporación de la metodología A+S a una actividad curricular, así como también incluir algún mecanismo de reconocimiento de las buenas prácticas en este tipo de cursos por parte de la unidad académica o de la universidad. Así mismo, surgen como desafíos la necesidad de realizar estudios desde la experiencia de implementación con los actores de este proceso formativo que interaccionan en el terreno social, a saber, supervisores docentes y de los socios comunitarios, estudiantes y usuarios idealmente en base a metodologías cualitativas que permitan comprender en profundidad y desde sus protagonistas los aportes y desafíos de la implementación de A+S en la formación de pregrado en Psicología en Chile.

\section{Referencias bibliográficas}

CLAYSS. (2016). Manual para docentes y estudiantes solidarios (4th ed.; Centro Latinoamericano de Aprendizaje y Servicio Solidario, ed.). Recuperado de http://www.clayss.org.ar/04_publicaciones/manual_docentes_LATAM.pdf

CNED. (2018). Las competencias para un munbdo en constante cambio: Habilidades del Siglo XXI. Ceremonia Celebración Día de Profesor Civil de La Armada de Chile, 1-25. Recuperado de https://www.cned.cl/sites/default/files/conferencia_armada_16-102018.pd

Elmore, R. (2010). Mejorando la escuela desde la sala de clases. Área de Educación Fundación Chile: Salesianos Impresores. 
Fadel, C., Bialik, M. \& Trilling, B. (2016). Educación en cuatro dimensiones. Las competencias que los estudiantes necesitan para su realización. Traducción Centro de Innovación en Educación de Fundación Chile. Recuperado de http://www.ccplm.cl/redcineclubescolar/wp-content/uploads/fadel-educacion-en-cuadrodimensiones.pdf

Fuentes, S \& Rosario, P. (2013). Mediar la autorregulación Del Aprendizaje: Un desafío para el siglo XXI (INDESCO \& Facultad de Ciencias de la Educación. Universidad Central de Chile, eds.). $\quad$ Recuperado de http://www.ucentral.cl/prontus_ucentral2012/site/artic/20130604/asocfile/20130604133302/ ebook_seminario__ara_julio_13_definitivo.pdf

González, E., Herrera, R., \& Zurita, R. (2008). Formación Basada en Competencias: Desafíos y Oportunidades. In CINDA, GRUPO OPERATIVO DE UNIVERSIDADES CHILENAS, \& MINEDUC (Eds.), Diseño curricular basado en competencias y aseguramiento de la calidad en la educación superior (pp. 375-386). Recuperado de https://www.cinda.cl/download/libros/39.pdf

Hodge, K. A., \& Lear, J. L. (2011). Employment Skills for 21st Century Workplace: The Gap Between Faculty and Student Perceptions. Journal of Career and Technical Education, 26(2), 28-41. https://doi.org/10.21061/jcte.v26i2.523

Jouannet, C., Salas, M. H., \& Contreras, M. A. (2013). Modelo de implementación de Aprendizaje Servicio $(A+S)$ en la UC: Una experiencia que impacta positivamente en la formación profesional integral. Calidad En La Educación, (39), 197-212. https://doi.org/10.4067/s0718-45652013000200007 
Martí, J. A.; Heydrich, M.; Rojas, M.; Hernández, A. (2010) Aprendizaje basado en proyectos: una experiencia de innovación docente. Revista Universidad EAFIT, vol. 46 (158), 11-21. Retrieved from https://www.redalyc.org/pdf/215/21520993002.pdf

Medina, E. (2010). Sergio Tobón Tobón. Formación integral y competencias. Pensamiento complejo, currículo, didáctica y evaluación. Revista Interamericana de Educación de $\begin{array}{lllll}\text { Adultos, } & 32 & \text { (2), } & \text { 90-95. } & \text { Recuperado }\end{array}$ https://www.redalyc.org/pdf/4575/457545095007.pdf

Ministerio de educación. (2007). Manual de aprendizaje Servicio. Recuperado de https://poligonosurelbarrioestuescuela.files.wordpress.com/2011/10/manual_aprendizaje_se rvicio.pdf

Opazo, H. (2015). Experiencias de aprendizaje- servicio en la formación del profesorado: Un estudio de caso (Tesis doctoral inédita). Universidad Autónoma de Madrid, España. Retrieved from https://repositorio.uam.es/handle/10486/670908

Scott, C. (2015). El futuro del aprendizaje 2 ¿Qué tipo de aprendizaje se necesita en el siglo XXI? Investigación y Prospectiva en Educación UNESCO, París. [Documentos de Trabajo ERF, No. 14]. Recuperado de https://unesdoc.unesco.org/ark:/48223/pf0000242996_spa

Recepción: 25 de noviembre de 2019

Última Revisión: 16 de diciembre 2019

Aceptación Final: 26 de diciembre 2019 